\section{Qualidade do ambiente e fatores \\ associados: um estudo em crianças de Pelotas, Rio Grande do Sul, Brasil}

\author{
Quality of the environment and associated \\ factors: a pediatric study in Pelotas, \\ Rio Grande do Sul, Brazil
}

Maria de Fátima Duarte Martins ${ }^{1}$
Juvenal Soares Dias da Costa ${ }^{2}$
Enrique Teófilo Saforcada 3
Melissa Dias da Costa Cunha ${ }^{4}$

\footnotetext{
1 Centro de Atendimento

à Saúde Escolar, Secretaria Municipal de Saúde

e Bem-Estar. Pelotas, Brasil.

2 Departamento de Medicina

Social, Faculdade de

Medicina, Universidade

Federal de Pelotas.

Pelotas, Brasil.

3 Facultad de Psicología,

Universidad de Belgrano.

Buenos Aires, Argentina.

4 Faculdade de Medicina,

Universidade Federal de

Pelotas. Pelotas, Brasil.

Correspondência Maria de Fátima Duarte Martins

Rua Marechal de Deodoro,

588/1103, Pelotas, RS

96020-220, Brasil.

neiam@hotmail.com
}

\section{Abstract}

This study aims to describe and identify factors associated with environmental quality and characteristics of children exposed to environmental risk factors in Pelotas, Rio Grande do Sul, Brazil. This was a prospective, populationbased study, including 630 children from the 1993 birth cohort. During the year 1998, environmental quality and other information were assessed using the Home Observation for the Measurement of the Environment. Data were submitted to univariate analysis. The association between the variables and the outcome was evaluated through prevalence ratios, 95\% confidence intervals, and chi-square. Logistic regression was performed according to a hierarchical model. Some 97 children (15\%) were living in negative environments. Eight risk factors were associated with environmental quality: low monthly family income, low maternal schooling, male gender, households with more than 7 members, 4 or more siblings, tobacco use during gestation, children sleeping in their parents' bed at age 4 years, and mothers with psychiatric disorders.

Environmental Quality; Risk Factors; Child Development; Educational Status

\section{Introdução}

Nos últimos 40 anos, investigações sobre o comportamento humano e o desenvolvimento infantil através de estudos antropológicos, sociológicos e psicológicos têm tentado responder a questões que se encontravam pendentes, estabelecendo uma forte dependência entre o ambiente da criança, sua saúde e seu desenvolvimento 1 . A literatura atual tem demonstrado que os cuidados prestados às crianças são conseqüências de muitos fatores, incluindo cultura, nível socioeconômico, estrutura familiar e características próprias da criança 2 .

Esse novo período é marcado pelo interesse em estudos sobre a relação mãe-filho e sobre a influência do ambiente no desenvolvimento das crianças. Autores como Belsky et al. 3 passaram a considerar a organização do ambiente físico e o entorno da criança como indicadores para o ótimo desenvolvimento de sua saúde. A psicologia passa a dar menos ênfase aos diagnósticos das psicopatologias e a se preocupar mais com os aspectos preventivos, com os programas de promoção de saúde e com a qualidade de vida das pessoas. As investigações sobre o desenvolvimento infantil, assim como os estudos clínicos, passam a incluir a avaliação do ambiente 4 .

A maneira pela qual os pais organizam o ambiente físico e interagem com os filhos tem influência sobre seu desenvolvimento. Desde 
que apoiada sobre bases biológicas e psicológicas suficientes e favoráveis para um desenvolvimento normal, essa criança poderá contar com um potencial de resiliência diante das adversidades físicas ou psicológicas. Se a criação não tiver ocorrido em um ambiente favorável, é provável que a criança apresente bases para a vulnerabilidade de seu desenvolvimento 5 .

Existe na literatura uma constante associação entre a qualidade do ambiente e o desenvolvimento psicológico das crianças, e a importância de fatores de risco biológicos e sociais para o desenvolvimento infantil. Baixo peso ao nascer, desnutrição, baixa renda familiar, baixa escolaridade dos pais, pais adolescentes, mães solteiras, ausência do pai, depressão materna, problemas psiquiátricos dos pais, famílias muito numerosas são alguns fatores de risco que se relacionam com a qualidade do ambiente e o desenvolvimento psicológico infantil 5,6,7 .

Apesar da importância do tema, foram encontrados poucos estudos dedicados à avaliação da qualidade do ambiente familiar com crianças brasileiras 8 . O presente estudo procurou identificar fatores de risco que possam estar associados à qualidade do ambiente e a algumas características das crianças expostas, e dessa forma contribuir como um aporte ao estudo do desenvolvimento infantil no que diz respeito a grupos vulneráveis, priorizando as ações protetoras de saúde.

Identificadas as variáveis associadas a ambientes negativos, é possível programar ações orientadas para os principais problemas de saúde de uma população específica, visando transferir os resultados para programas de saúde coletiva, privilegiando a proteção e promoção da saúde, a fim de evitar o crescimento exagerado das instituições e dos serviços, com uma demanda crescente e descontrolada que engrossa a taxa de morbidade da sociedade brasileira.

\section{Materiais e métodos}

Em 1993, a equipe de investigadores do Departamento de Medicina Social da Universidade Federal de Pelotas, Rio Grande do Sul, Brasil, iniciou um estudo longitudinal que acompanhou todos os nascimentos hospitalares ocorridos na cidade de Pelotas, Rio Grande do Sul, Brasil 9.

De 1o de janeiro a 31 de dezembro de 1993, nasceram em hospitais públicos e privados da cidade 5.304 crianças. Essas crianças integraram um estudo de coorte e as diversas fases de seu desenvolvimento vêm sendo acompanhadas.

O estudo longitudinal teve uma coordenação central, tendo os outros projetos coordenação própria. Os sujeitos do estudo foram 634 crianças e respectivas mães, contatadas no ano de 1997 para participar desse acompanhamento 10 .

Nesse ano, um novo projeto foi realizado com o objetivo de avaliar a qualidade do entorno das crianças. Foram encontradas 1.273 crianças que fazem parte da coorte de 1993 e, por meio de uma seleção sistemática - uma criança sim, outra não, segundo uma ordem cronológica -, metade dessa submostra $(n=634)$ foi recrutada para participar do novo estudo. As crianças apresentavam idade média de 4 anos e 5 meses $(\mathrm{dp}=3,60)$.

Para essa investigação, foram usados três questionários: (1) o questionário perinatal (1993) foi aplicado à mãe no hospital, com o objetivo de examinar sua história reprodutiva, fatores demográficos e sócio-econômicos; (2) o questionário dos 4 anos (1998), primeira visita, foi aplicado com o objetivo de convidar a criança para participar do novo estudo e, após consentimento familiar, coletar informações sobre coleito e a cor da criança; (3) o questionário dos 4 anos (1998), segunda visita, foi respondido pela mãe e buscou dados sobre a configuração familiar. Durante a segunda visita foram aplicados o Home Observation for the Measurement of the Environment (HOME) e o Self-Reported Questionnaire of Minor Psychiatric Disorders (SRQ-20). Em todas as fases foram aplicados questionários padronizados, pré-codificados e previamente testados.

Para avaliar a qualidade e a quantidade dos estímulos oferecidos pelo ambiente tomado como variável dependente, utilizou-se o HOME 11,12. O HOME é um instrumento concebido para medir a qualidade e a quantidade de apoio social, emocional e cognitivo disponível para a criança em seu ambiente familiar. Foi utilizada a versão $M C$-HOME para pré-escolares. O instrumento, composto de 55 itens, tem demonstrado capacidade adequada para avaliar a qualidade do ambiente em diferentes estratos sociais e para detectar características familiares quando aplicado em populações latino-americanas 5,13. O HOME compreende uma entrevista com a mãe e a observação direta da interação entre mãe e filho. Os instrumentos foram aplicados nas casas das crianças, quando estas estavam acordadas e na presença das mães ou 
de cuidadores. Os itens foram codificados em termos de ambientes bons (positivos) e ambientes ruins (negativos). A qualidade insuficiente do $H O M E$ correspondia ao escore $<30$.

Foram coletadas informações sobre outras variáveis tomadas como possíveis associações com a qualidade do ambiente. Essas variáveis foram escolhidas de acordo com a existência de antecedentes na literatura

As variáveis sócio-econômicas e familiares foram renda familiar mensal em salários mínimos e número de pessoas na residência. Como variáveis maternas foram incluídas idade, escolaridade em anos de estudo, uso de tabaco e consumo de bebida alcoólica durante a gestação, situação marital, além da presença de distúrbios psiquiátricos menores.

Para avaliar os transtornos psiquiátricos menores das mães utilizou-se o $S R Q-20$ 14. O $S R Q-20$ foi concebido para detectar psicopatologias psiquiátricas em atenção primária à saúde com fins de investigação e não de diagnóstico. O questionário é composto de 20 itens. Utilizou-se a versão de Mary \& Williams 15, validada para o Brasil. O ponto de corte estabelecido para caracterizar a presença de distúrbios psiquiátricos menores foi de 7 para as mulheres.

As variáveis paternas incluídas no estudo foram idade e escolaridade. As variáveis relacionadas com as crianças foram sexo, cor da pele, número de irmãos e coleito aos 4 anos.

O trabalho de campo começou em janeiro de 1998 e foi concluído em abril do mesmo ano. Das 634 crianças encontradas, houve quatro perdas da mostra original: duas por mudança de endereço e duas por se recusarem a prosseguir no estudo. Portanto, a amostra final foi integrada por 630 crianças.

O trabalho de campo foi realizado pela equipe de entrevistadores, constituída de seis psicólogos responsáveis pela aplicação dos instrumentos, uma supervisora de campo, uma coordenadora geral e duas suplentes.

Todos os questionários foram revisados pela supervisora de campo. A entrada de dados foi realizada duas vezes através do programa Epi-Info. O controle de qualidade foi feito em $5 \%$ da amostra, selecionada aleatoriamente.

A confiabilidade do HOME foi medida com o alfa de CRONBACH, com um resultado de $\mu=$ 0,998 para as variáveis padronizadas. Para as variáveis não padronizadas o resultado foi praticamente igual, recém no quarto dígito decimal se observa uma pequena diferença. Portanto, pode-se considerar que existe alta confiabilidade. Para verificar o grau de correlação entre as variáveis, utilizou-se o teste de correlação de Spearman. O resultado demonstrou que o HOME, quando se analisam as correlações de Spearman, constata que as relações entre as distintas variáveis que o compõem são baixas, o que significa que cada variável está medindo alguma dimensão diferente do sujeito avaliado. A correlação média interitens da escala foi de $\mathrm{r}^{2}=0,981$, considerada muito alta. Logo, pode-se afirmar que o HOME possui alta confiabilidade e todos os itens da escala medem aspectos do constructo.

Para a análise estatística dos dados utilizou-se o programa SPSS para Windows.

A análise univariada foi utilizada para obter a freqüência de todas as variáveis de interesse e examinou sua distribuição. A análise bivariada foi realizada com cada variável independente em relação ao inventário $H O M E$, através de tabelas de contingência para a análise do $c h i$ square de Pearson e teste de tendência linear.

Para a análise multivariada foram selecionadas todas as variáveis que estiveram associadas a ambientes negativos com $\mathrm{p}<0,20$. Para esta análise usou-se o modelo hierárquico (Figura 1). As variáveis nos níveis 1,2 e 3 são as variáveis independentes o HOME constituí a variável dependente. Os valores desta variável são 1 e 0 , ou seja, é uma variável dicotômica, situação avaliada pela análise estatística. Através da regressão logística calcularam-se as razões de odds e seus intervalos de confiança.

Calcularam-se as razões de prevalência para ambientes negativos em um intervalo de confiança de $95 \%$.

\section{Resultados}

A amostra é composta de 630 crianças de ambos os sexos, distribuídos de forma semelhante, com $50,40 \%$ correspondendo a meninas. As crianças eram predominantemente de cor branca $(76,40 \%)$. Das crianças visitadas, 97 (15,40\%; IC95\% 12,60-18,20) foram encontradas vivendo em ambientes de baixa estimulação e apoio, classificados como negativos pelo HOME.

A Tabela 1 apresenta a distribuição da amostra conforme as características socioeconômicas e familiares, maternas, paternas e relacionadas com as crianças.

A principal categoria de renda familiar situou-se entre 3,10 e 6,00 salários-mínimos $(30,50 \%)$. Cerca de $15 \%$ das crianças viviam em casas com sete pessoas ou mais. A idade média das mães quando nasceram as crianças era de 31 anos ( $d p=6,40)$, sendo que $13 \%$ das mães eram adolescentes (menos de 20 anos). Aproximadamente $28 \%$ das mães (172) completaram até quatro anos de escolaridade e, 
destas, 14 (2,30\%) não completaram nem um ano. Em relação ao comportamento materno durante a gravidez, $31 \%$ referiram não usar tabaco e 4,90\% ingeriram bebida alcoólica. Quase $90 \%$ das mulheres viviam com o marido ou companheiro.

Os pais tinham em média 34 anos de idade $(\mathrm{dp}=7,60)$ e aproximadamente $7 \%$ tinham 20 anos ou menos. Cerca de $4 \%$ dos pais eram analfabetos, e a escolaridade média foi de sete anos $(\mathrm{dp}=3,70)$. Aproximadamente $10 \%$ das crianças possuíam quatro ou mais irmãos. Cerca de $28 \%$ dormiam na cama dos pais aos 4 anos de idade. Os transtornos psiquiátricos menores correspondentes a estresse psicológico foram encontrados em um quarto das mulheres do estudo.

Em relação às variáveis sócio-econômicas e familiares, verificou-se que a qualidade do ambiente estava associada com a renda mensal. À medida que diminuíam os níveis de renda, aumentava o percentual de ambientes negativos (teste de tendência linear $<0,01$ ). Observou-se que as residências com sete ou mais pessoas também apresentavam maior probabilidade de serem classificadas como negativas (Tabela 1).

Quanto às variáveis maternas, os dados revelaram uma associação significativa para escolaridade materna e qualidade do ambiente. Constatou-se que, quanto menor a categoria de escolaridade, maior a probabilidade de ambientes negativos (teste de tendência linear $<0,01$ ). Da mesma forma, as mães que apresentavam distúrbios psiquiátricos menores contribuíam para a prevalência de ambientes negativos. Foi encontrada uma associação quanto ao uso de tabaco na gestação e ambientes negativos. Em relação à distribuição por idade, consumo de álcool e presença de marido ou companheiro não foram encontradas diferenças significativas (Tabela 1).

Entre as variáveis paternas, foram encontradas diferenças estatisticamente significativas para escolaridade. A prevalência de ambientes negativos aumentava à medida que diminuíam as categorias de escolaridade (teste de tendência linear $<0,01$ ). Não foi encontrada associação entre a idade paterna e a qualidade do ambiente (Tabela 1).

Nas variáveis relacionadas com as características das crianças, foram encontradas diferenças estatisticamente significativas quanto ao sexo: $11,60 \%$ das meninas contra $19,20 \%$ dos meninos viviam em ambientes negativos $(\mathrm{p}<0,01)$. Os ambientes negativos estavam associados a quatro ou mais irmãos e à prática de dormir com os pais. Não foram encontradas diferenças estatisticamente significativas em relação à cor da pele (Tabela 1).
$\mathrm{Na}$ análise de regressão logística, foram selecionadas todas as variáveis que estiveram associadas à ocorrência de ambientes negativos com $\mathrm{p}<0,20$. Para esta análise foi utilizado um modelo hierárquico (Figura 1). Para cada nível foram introduzidas todas as variáveis selecionadas e, independentemente da modificação de seus valores, sempre foram mantidas no modelo as variáveis que entraram em um nível hierárquico superior. Após as análises, permaneceram associados a renda familiar mensal: escolaridade materna, sexo das crianças, número de irmãos, famílias com mais de sete pessoas, uso de tabaco na gestação, coleito aos 4 anos e presença de distúrbios psiquiátricos menores (Tabela 2). Essas oito variáveis foram consideradas fatores de risco associados à qualidade do ambiente.

\section{Discussão}

Muitas características da criança e da família, bem como o contexto social, podem expor a criança a sérios problemas no futuro. Os estudos mais recentes sobre o desenvolvimento infantil enfatizam a importância de se examinar o contexto em que este ocorre e em especial o efeito da presença simultânea de múltiplos fa-

Figura 1

Modelo análise hierarquizado

Renda familiar, idade da mãe, escolaridade materna,
cor e sexo da criança

Nível 2 Número de pessoas no domicílio, situação marital, uso de tabaco na gestação, consumo de álcool na gestação, número de irmãos, coleito

Nível $3 \quad$ Transtornos psiquiátricos menores na mãe

Ambientes positivos 
Características da população estudada, percentuais de distribuição quanto à presença de ambiente positivo, razões de prevalência e respectivos intervalos de confiança. Pelotas, Rio Grande do Sul, Brasil, 1998.

\begin{tabular}{|c|c|c|c|c|c|}
\hline \multirow[t]{2}{*}{ Variável } & \multicolumn{3}{|c|}{ Ambiente positivo } & \multirow{2}{*}{$\begin{array}{l}\text { Razão de } \\
\text { prevalência }\end{array}$} & \multirow[t]{2}{*}{ IC95\% } \\
\hline & $\mathrm{n}$ & $n$ & $\%$ & & \\
\hline \multicolumn{6}{|c|}{ Renda familiar mensal (em salários mínimos) } \\
\hline 6,1 ou mais & 143 & 137 & 95,80 & 1,00 & \\
\hline $3,1-6,0$ & 188 & 168 & 89,40 & 2,55 & $1,05-6,15$ \\
\hline $1,1-3,0$ & 190 & 161 & 84,70 & 3,64 & $1,55-8,30$ \\
\hline$<1$ & 96 & 56 & 58,30 & 9,93 & $4,38-22,51$ \\
\hline \multicolumn{6}{|c|}{ Número de pessoas } \\
\hline Menos de 7 & 587 & 508 & 86,50 & 1,00 & - \\
\hline 7 ou + & 43 & 25 & 58,10 & 3,11 & $2,07-4,68$ \\
\hline \multicolumn{6}{|c|}{ Idade materna (anos) } \\
\hline$<20$ & 84 & 72 & 83,70 & 1,00 & - \\
\hline $20-24$ & 159 & 133 & 83,60 & 1,14 & $0,61-2,15$ \\
\hline $25-29$ & 168 & 141 & 83,90 & 1,13 & $0,60-2,11$ \\
\hline $30-35$ & 140 & 126 & 90,00 & 0,70 & $0,34-1,44$ \\
\hline $36-39$ & 60 & 49 & 79,00 & 1,47 & $0,72-2,99$ \\
\hline 40 ou + & 18 & 13 & 72,20 & 1,94 & $0,78-4,83$ \\
\hline \multicolumn{6}{|c|}{ Escolaridade materna (anos) } \\
\hline 9 ou + & 176 & 171 & 97,20 & 1,00 & - \\
\hline $5-8$ & 275 & 244 & 88,70 & 3,97 & $1,57-10,01$ \\
\hline $1-4$ & 158 & 109 & 69,00 & 10,92 & $4,46-26,71$ \\
\hline 0 & 147 & 5 & 35,70 & 22,63 & $8,77-58,40$ \\
\hline \multicolumn{6}{|c|}{ Uso de tabaco na gestação } \\
\hline Não & 435 & 384 & 88,30 & 1,00 & - \\
\hline Sim & 195 & 149 & 76,40 & 2,01 & $1,40-2,89$ \\
\hline \multicolumn{6}{|c|}{ Consumo de álcool } \\
\hline Não & 599 & 507 & 84,60 & 1,00 & - \\
\hline Sim & 31 & 26 & 83,90 & 1,05 & $0,46-2,40$ \\
\hline \multicolumn{6}{|c|}{ Vive com marido ou companheiro } \\
\hline Não & 565 & 480 & 85,00 & 1,00 & - \\
\hline Sim & 65 & 53 & 81,50 & 1,23 & $0,71-2,12$ \\
\hline \multicolumn{6}{|c|}{ Distúrbios psiquiátricos menores } \\
\hline Não & 465 & 411 & 88,40 & 1,00 & - \\
\hline Sim & 159 & 116 & 73,00 & 2,33 & $1,63-3,33$ \\
\hline \multicolumn{6}{|c|}{ Idade paterna (anos) } \\
\hline$\leq 20$ & 47 & 40 & 85,10 & 1,00 & - \\
\hline $21-24$ & 113 & 98 & 86,70 & 0,89 & $0,39-2,04$ \\
\hline $25-29$ & 158 & 134 & 84,80 & 1,02 & $0,47-2,22$ \\
\hline $30-34$ & 146 & 127 & 87,00 & 0,87 & $0,39-1,95$ \\
\hline $35-39$ & 81 & 70 & 86,40 & 0,91 & $0,38-2,19$ \\
\hline $40-44$ & 47 & 39 & 83,00 & 1,14 & $0,45-2,90$ \\
\hline 45 ou + & 29 & 19 & 65,50 & 2,32 & $0,99-5,40$ \\
\hline
\end{tabular}




\begin{tabular}{|c|c|c|c|c|c|}
\hline \multirow{2}{*}{ Variável } & \multicolumn{3}{|c|}{ Ambiente positivo } & \multirow{2}{*}{$\begin{array}{l}\text { Razão de } \\
\text { prevalência }\end{array}$} & \multirow[t]{2}{*}{ IC95\% } \\
\hline & $\mathrm{n}$ & $\mathrm{n}$ & $\%$ & & \\
\hline \multicolumn{6}{|c|}{ Escolaridade paterna (anos) } \\
\hline 9 ou + & 176 & 150 & 96,80 & 1,00 & - \\
\hline $5-8$ & 288 & 248 & 86,10 & 4,31 & $1,73-10,69$ \\
\hline $1-4$ & 121 & 98 & 81,00 & 5,89 & $2,31-15,05$ \\
\hline 0 & 22 & 7 & 31,80 & 21,14 & $8,52-52,42$ \\
\hline \multicolumn{6}{|c|}{ Sexo das crianças } \\
\hline Feminino & 318 & 281 & 88,40 & 1,00 & - \\
\hline Masculino & 312 & 252 & 80,80 & 1,65 & $1,13-2,41$ \\
\hline \multicolumn{6}{|l|}{ Cor da pele } \\
\hline Branca & 478 & 417 & 87,20 & 1,00 & - \\
\hline Não branca & 148 & 112 & 75,70 & 1,91 & $1,32-2,75$ \\
\hline \multicolumn{6}{|c|}{ Número de irmãos } \\
\hline$<4$ & 571 & 503 & 88,10 & 1,00 & - \\
\hline$\geq 4$ & 60 & 31 & 51,70 & 4,06 & $2,88-5,72$ \\
\hline \multicolumn{6}{|c|}{ Coleito aos 4 anos } \\
\hline Não & 456 & 407 & 89,30 & 1,00 & $1,87-3,80$ \\
\hline $\operatorname{Sim}$ & 171 & 122 & 71,30 & 2,67 & - \\
\hline
\end{tabular}

tores de risco, tanto biológicos como ambientais. Estudos constataram que a variável de maior impacto sobre o desenvolvimento infantil é a estimulação do ambiente 5,8. Portanto, avaliar a estimulação disponível para a criança dentro de uma determinada família, pode fornecer elementos importantes para as políticas de saúde e educação a serem programadas pelos organismos públicos.

Este estudo constatou que as famílias de baixo ingresso estão mais expostas a ambientes negativos. A renda familiar é determinante para a qualidade de vida das famílias quanto ao acesso a saúde, educação, alimentação e habitação, entre outros. As dificuldades constantes associadas à pobreza prejudicam o bem-estar psicológico dos pais e o ambiente interpessoal na casa, e é possível afirmar que a boa qualidade da criação dos filhos requer gastos consideráveis em investimentos por parte da família que favoreçam o seu desenvolvimento. Embora o resultado encontrado seja consistente com outros estudos, de acordo com Bee 16, mesmo reconhecendo que há variáveis comportamentais relacionadas com o status socioeconômico, não se pode legitimamente inferir o potencial estimulador do ambiente familiar a partir apenas do conhecimento da classe social. Ainda de acordo com o autor, os grupos socioeconômicos inferiores apresen- tam a mesma amplitude de práticas de criação de filhos e atitudes encontradas nos grupos socioeconômicos médios. Acima de uma boa renda familiar, uma relação positiva entre pais e filhos, construída sobre uma base de amor, pode aumentar a competência social da criança e sua disposição para explorar e se arriscar.

As mães com maior escolaridade alcançaram menores percentuais de ambientes negativos. Pode-se supor que as mães com escolaridade tiveram mais acesso a informações sobre desenvolvimento infantil e que desta forma interagem melhor com seus filhos, respondem adequadamente às suas solicitações e podem prover melhores condições físicas e emocionais para o desenvolvimento de seu filho. Embora os resultados do estudo tenham encontrado uma forte associação entre a escolaridade materna e a qualidade do ambiente, mais do que o grau de instrução materno, o comportamento afetivo, a estabilidade no relacionamento e um bom nível de cuidados com a criança são fatores de proteção e enriquecimento para o desenvolvimento infantil.

Não se encontrou associação entre a idade da mãe no momento do nascimento e a qualidade do ambiente. Constatou-se, porém, que as mães com idade entre 30 e 35 anos (não adolescentes) apresentaram menor número de ambientes negativos. Para Dubow \& Luster 17, 
Tabela 2

Análise de regressão logística dos fatores de risco para ambientes negativos. Pelotas, Rio Grande do Sul, Brasil, 1993 e 1998.

\begin{tabular}{|c|c|c|c|}
\hline Variável & Odds ratio & $\begin{array}{c}\text { Intervalo de } \\
\text { confiança } 95 \%\end{array}$ & P-valor \\
\hline \multicolumn{4}{|c|}{$\begin{array}{l}\text { Renda familiar mensal } \\
\text { (em salários mínimos) }\end{array}$} \\
\hline 6,1 ou + & 1,00 & - & - \\
\hline $3,1-6,0$ & 1,24 & $0,45-3,40$ & - \\
\hline $1,1-3,0$ & 1,55 & $0,58-4,17$ & - \\
\hline$<1$ & 4,59 & $1,68-12,55$ & $<0,001$ \\
\hline \multicolumn{4}{|c|}{ Escolaridade materna (anos) } \\
\hline 9 ou + & 1,00 & - & \\
\hline $5-8$ & 3,35 & $1,24-9,08$ & - \\
\hline $1-4$ & 9,40 & $3,42-25,81$ & - \\
\hline 0 & 23,57 & $5,17-107,46$ & \\
\hline \multicolumn{4}{|l|}{$<0,0001$} \\
\hline \multicolumn{4}{|c|}{ Sexo das crianças } \\
\hline Feminino & 1,00 & - & - \\
\hline Masculino & 2,26 & $1,36-3,78$ & $<0,01$ \\
\hline \multicolumn{4}{|c|}{ Número de pessoas* } \\
\hline Menos de 7 & 1,00 & - & - \\
\hline $7 \mathrm{ou}+$ & 2,15 & $0,91-5,06$ & 0,08 \\
\hline \multicolumn{4}{|c|}{ Número de irmãos* } \\
\hline$<4$ & 1,00 & - & - \\
\hline$\geq 4$ & 2,91 & $1,38-6,13$ & $<0,01$ \\
\hline \multicolumn{4}{|c|}{ Uso de tabaco na gestação* } \\
\hline Não & 1,00 & - & - \\
\hline Sim & 2,27 & $1,33-3,88$ & $<0,01$ \\
\hline \multicolumn{4}{|c|}{ Coleito aos 4 anos* } \\
\hline Não & 1,00 & - & - \\
\hline Sim & 2,66 & $1,55-4,56$ & $<0,001$ \\
\hline \multicolumn{4}{|c|}{ Distúrbios psiquiátricos menores ${ }^{\star \star}$} \\
\hline Não & 1,00 & - & - \\
\hline Sim & 1,81 & $1,04-3,14$ & 0,05 \\
\hline
\end{tabular}

* Ajustados para renda familiar mensal, escolaridade materna, sexo das crianças. ** Ajustados para renda familiar mensal, escolaridade materna, sexo das crianças, número de pessoas, número de irmãos, uso de tabaco na gestação,

coleito aos 4 anos. tregavam seus bebês para adoção e seus filhos tendiam a obter baixos resultados nos testes cognitivos quando comparados aos de mães não adolescentes. É possível que as mães do presente estudo tenham um bom suporte familiar emocional e financeiro que lhes permitam prover um ambiente de boa qualidade e amor para seus filhos. A adolescência é uma fase do crescimento com especificidades e vulnerabilidades próprias. A gravidez nessa fase da vida é cada vez mais freqüente e ocorre, em geral, em momentos de indefinição, indecisão e falta de conhecimento sobre educação sexual, sexualidade e planejamento familiar. Portanto, a prevenção à gravidez na adolescência é um tema que deve fazer parte dos programas de saúde.

A presença do pai ou substituto não teve, neste estudo, efeito sobre a qualidade do ambiente. Para Bradley \& Whiteside-Mansell 18, Caplan 19 e Rutter 20, as discórdias maritais e as discussões constantes do casal na frente da criança a atingem diretamente, sendo provável que sejam mais determinantes na qualidade do ambiente do que a ausência de marido ou companheiro. Talvez as mães aqui avaliadas, que vivem sem companheiro, ofereçam um bom suporte social, sejam fontes de afeto, afirmação e apoio para seus filhos e que estes atributos funcionem como protetores contra os efeitos negativos.

O consumo de bebida alcoólica durante a gravidez não esteve associado a ambientes negativos. De acordo com a literatura, crianças que vivem em famílias com alcoólatras tendem a ser hiperativas, a ter baixa auto-estima e problemas escolares, além do risco de desenvolver a doença eles próprios 21. É possível que no presente estudo as mães não tenham declarado o uso de álcool durante a gravidez por conhecerem os possíveis riscos para a criança. Um resultado diferente poderia ser esperado se esta variável fosse coletada durante a fase de avaliação do ambiente, pois há evidências de comportamento anti-social e desorganização familiar nos alcoólatras.

O fumo durante a gravidez esteve associado com a qualidade do ambiente, mas não se encontraram estudos que avaliaram a associação deste hábito com a qualidade do ambiente. No entanto, estão bem documentados outros malefícios provocados pelo consumo de tabaco durante a gestação, tais como maior incidência de baixo peso no nascimento e retardo do crescimento intra-uterino 22 .

Encontrou-se uma significativa relação entre a presença de transtornos psiquiátricos menores na mãe e a qualidade do ambiente. As mães que tiveram menores indicadores de sus- 
peita de transtornos psiquiátricos, de acordo a avaliação do $S R Q-20$, apresentaram ambientes positivos. A hipótese de que a saúde mental da mãe influencia o desenvolvimento infantil tem orientado um número significativos de estudos $6,23,24$. As doenças mentais maternas que demonstraram afetar a conduta das crianças são a esquizofrenia e a depressão. Mães depressivas têm dificuldade de interagir com seu bebê, são mais negativas e apresentam maior dificuldade de interação social, colocando a criança em risco de desenvolver problemas afetivos.

O presente estudo analisou algumas características das crianças que convivem em ambientes negativos. Como em outros estudos, encontrou-se uma prevalência de meninos nesses ambientes. O estudo de Bastos \& Almeida Filho ${ }^{8}$, realizado no Brasil, revelou que o sexo masculino estaria mais exposto a riscos. As meninas apresentavam maior vulnerabilidade diante da influência de um ambiente doméstico mais restrito, enquanto os meninos estavam expostos a uma estimulação mais ampla, tanto social quanto cultural. Nesse estudo, casas com famílias numerosas e a presença de mais de quatro irmãos na mesma residência estiveram associados à qualidade do ambiente. Outros estudos, como o de Sameroff et al. 25 e de Andraca et al. 5, consideraram essa variável como fator de risco para a qualidade do ambiente, pois famílias numerosas tendem a ser menos estimuladoras.

O presente estudo revelou que crianças que dormem na cama dos pais aos 4 anos de idade estão mais presentes em domicílios com quali- dade do ambiente negativa. De acordo com Lozoff \& Davis 26, essas crianças tornam-se mais vulneráveis a problemas emocionais e comportamentais, e apresentam maus resultados nas atividades que exigem concentração e atenção.

Os resultados acima permitiram identificar padrões de comportamento por meio do conhecimento do contexto social e econômico de uma determinada população, através dos quais é possível delinear estratégias para enfrentar esses problemas.

É importante ressaltar que os programas vinculados a saúde infantil devem estar voltados para o ambiente da criança, pois é nele que ela se estrutura como um ser individual e social, capaz de interagir com outros níveis de amplitude e de construir espaços entre os setores sociais envolvidos no processo de construção de uma vida saudável. Segundo Winnicott 27, "tudo começa em casa". A família é o primeiro agrupamento, e de todos os agrupamentos é o que está mais próximo de ser um agrupamento dentro da unidade da personalidade.

Ocupar-se dessa população para que ela não adoeça, priorizar programas que protejam a saúde, chamar as famílias para participar ativamente do cuidado de sua saúde e a adoção de modos de vida saudáveis, além da percepção de riscos, são ações que podem diminuir o impacto de acontecimentos estressantes que essas crianças possam vir a sofrer quando expostas a conseqüências negativas no que se refere a questões de saúde, tanto em sua perspectiva pessoal-subjetiva como social-comunitária.

\section{Resumo}

Descrever e identificar fatores que possam estar associados à qualidade do ambiente e características das crianças expostas, em Pelotas, Rio Grande do Sul, Brasil. Trata-se de um estudo prospectivo de caráter populacional, incluindo 630 crianças da coorte de nascimentos de 1993. No ano de 1998 avaliou-se, entre outras informações, a qualidade do ambiente, medida através do Home Observation for the Measurement of the Environment. Os dados foram analisados por meio de análise univariada. A associação entre as variáveis e o desfecho foi avaliada por meio das razões de prevalência, dos intervalos de confiança em $95 \%$ e do qui-quadrado. Realizou-se a regressão logística se- guindo modelo hierarquizado. Constatou-se que 97 crianças (15\%) viviam em ambiente negativo. Encontraram-se oito fatores de risco associados à qualidade do ambiente: baixa renda familiar mensal, baixa escolaridade materna, sexo masculino, casas com mais de sete residentes, número de irmãos maior ou igual a quatro, uso de tabaco na gestação, crianças que dormem na cama dos pais aos 4 anos e mães com presença de transtornos psiquiátricos.

Qualidade do Ambiente; Fatores de Risco; Desenvolvimento Infantil; Escolaridade 


\section{Colaboradores}

M. F. D. Martins elaborou o artigo. J. S. D. Costa contribuiu na revisão do artigo. E. T. Saforcada participou da revisão do artigo. M. D. Costa digitou o artigo.

\section{Agradecimentos}

Ao Departamento de Medicina Social da Faculdade de Medicina da Universidade Federal de Pelotas nas pessoas de Cesar G. Victora e Fernando Barros. Ao professor Dr. Carlos Lac Prugent, vice-reitor da Universidad CAECE, Buenos Aires, Argentina, a Luciana Anselmi e Rosângela Lima.

\section{Referências}

1. Seifer R, Sameroff AJ, Barrett LC, Krafchuk E. Infant temperament measured by multiple observations and mother report. Child Dev 1994; 65: 1478-90.

2. Bronfenbrenner U. La ecologia del desarollo humano. Barcelona: Editora Paidós; 1987.

3. Belsky J, Garduque L, Herneir E. Assessing performance, competence and executive capacity in infant play: relations to home environment and security of attachment. Dev Psychol 1984; 20:406-17.

4. Grizenko N, Fisher C. Review of studies of risk and protective factors for psychopathology in children. Can J Psychiatry 1992; 37:711-21.

5. Andraca I, Pino P, La Parra A, Rivera F, Castilo M. Risk factors for psychomotor development among infants born under optimal biological conditions. Rev Saúde Pública 1998; 32:138-47.

6. Seifer R, Sameroff AJ, Dickstein S, Keitner G, Miller I, Rasmussen S. Parental psychopathology, multiple contextual risks, and one-year outcomes in children. J Clin Child Psychol 1996; 25:423-35.

7. Sameroff AJ, Seifer R, Baldwin A, Baldwin C. Stability of intelligence from preschool to adolescence: the influence of social and family risk factors. Child Dev 1993; 64:80-97.

8. Bastos ACS, Almeida NM. Variables económicosociales, ambiente familiar y salud mental infantil en un área urbana de Salvador (Bahía), Brasil. Acta Psiquiatr Psicol Am Lat 1990; 36:147-54.

9. Victora CG, Barros FC, Halpern R, Menezes AM, Horta BL, Tomasi E. Longitudinal study of the mother and child population in an urban region of Southern Brazil, 1993: methodological aspects and preliminary results. Rev Saúde Pública 1996; 30:34-45.

10. Anselmi L, Piccini CA, Barros FC, Victora CG, Lopes RS. Psychosocial determinants of behaviour problems in Brazilian preschool children. J Child Psychol Psychiatr 2003; 44:1-10.

11. Gottfried AW. Home environment and early cognitive development. California: Academic Press; 1984.

12. Mundfrom D, Bradley HR. A factor analytic study of the infant-toddler and early childhood versions of the home inventory. Educ Psychol Meas 1993; 53:479.

13. Lozoff B, Park AM, Radan AE, Wolf AW. Using the HOME inventory with infants in Costa Rica. Int J Behav Dev 1995;18:277-95.

14. Harding TW, Climent CE, Diop M, Giel R, Ibrahim $\mathrm{HH}$, Murthy RS, et al. The WHO collaborative

study on strategies for extending mental health care: the development of new research methods. Am J Psychiatry 1983; 140:1474-80.

15. Mari JJ, Williams P. Comparison of the validity of two psychiatric screening questionnaires (GHQ12 and SRQ-20) in Brazil, using Relative Operating Characteristic (ROC) analysis. Psychol Med 1985; 15:651-9.

16. Bee H. Psicologia do desenvolvimento: questões sociais. Rio de Janeiro: Editora Interamericana; 1979.

17. Dubow EF, Luster T. Adjustment of children born to teenage mothers: the contribution of risk and protective factors. J Marriage Fam 1990; 52:393404.

18. Bradley RH, Whiteside-Mansell L. Parents socioemotional investment in children. J Marriage Fam 1997; 59:77.

19. Caplan HL, Cogill SR, Alexandra H, Robson KM, Katz R, Kumar R. Maternal depression and the emotional development of the child. Br J Psychiatry $1989 ; 154: 818-22$.

20. Rutter M. Parents - child separation: psychological effects on the children. J Child Psychol Psychiatr 1971; 123:233-60.

21. Hill SY, Hruska DR. Childhood psychopathology in families with multigenerational alcoholism. J Am Acad Child Adolesc Psychiatry 1992; 31:102430.

22. Horta BL, Victora CG, Menezes AMB, Halpern R, Barros FC. Low birthweight, preterm births and intrauterine growth retardation in relation to maternal smoking. Pediatri Perinat Epidemiol 1997; 11:140-51.

23. Bastos ACS, Almeida Filho N. Determinação social da saúde mental infantil: revisão da literatura epidemiológica. Psicol Teor Pesqui 1988; 4:268-82.

24. Cummings EM, Davies PT. Maternal depression and child development. J Child Psychol Psychiatry $1994 ; 35: 73-112$.

25. Sameroff AJ, Seifer R. Familial risk and child competence. Child Dev 1983; 54:1254-68.

26. Lozoff B, Wolf B, Davis NS. Cosleeping in urban families in the United States. Pediatrics 1984; 74:171-82.

27. Winnicot DW. Tudo começa em casa. São Paulo: Livraria Martins Fontes Editora; 1989.

Recebido em 19/Nov/2002

Versão final reapresentada em 26/Set/2003

Aprovado em 09/Out/2003 